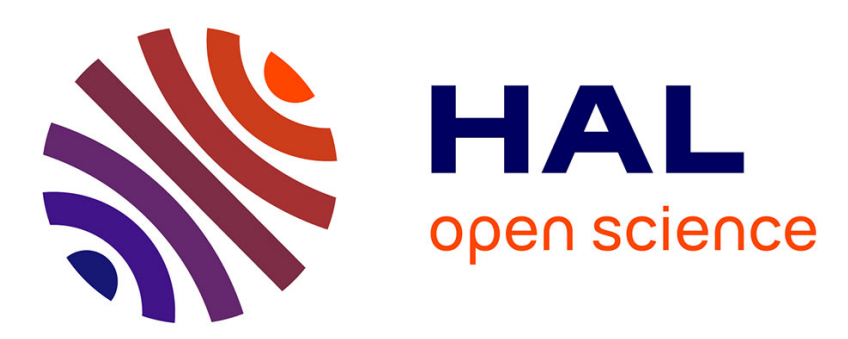

\title{
Ségrégation compétitive du soufre et du silicium sur la face (100) d'un alliage Fe-6\%at. Si
}

M. Essouni, M. F. Boudjemaa, A. Mosser

\section{To cite this version:}

M. Essouni, M. F. Boudjemaa, A. Mosser. Ségrégation compétitive du soufre et du silicium sur la face (100) d'un alliage Fe-6\%at. Si. Revue de Physique Appliquée, 1988, 23 (8), pp.1355-1360. 10.1051/rphysap:019880023080135500 . jpa-00245953

\section{HAL Id: jpa-00245953 https://hal.science/jpa-00245953}

Submitted on 1 Jan 1988

HAL is a multi-disciplinary open access archive for the deposit and dissemination of scientific research documents, whether they are published or not. The documents may come from teaching and research institutions in France or abroad, or from public or private research centers.
L'archive ouverte pluridisciplinaire HAL, est destinée au dépôt et à la diffusion de documents scientifiques de niveau recherche, publiés ou non, émanant des établissements d'enseignement et de recherche français ou étrangers, des laboratoires publics ou privés. 
Classification

Physics Abstracts

$64.75-81.30 \mathrm{M}$

\title{
Ségrégation compétitive du soufre et du silicium sur la face (100) d'un alliage Fe-6\%at. Si
}

\author{
M. Essouni, M. F. Boudjemaa et A. Mosser \\ I.P.C.M.S., Unité Mixte CNRS-ULP-EHICS n ${ }^{\circ}$ 3800486, Groupe Surfaces-Interfaces, 4 rue Blaise Pascal, \\ 67000 Strasbourg, France
}

(Reçu le 20 juillet 1987, révisé le 3 février 1988, accepté le 2 mai 1988)

\begin{abstract}
Résumé. - La ségrégation du soufre et du silicium sur la face (100) d'un alliage monocristallin de Fe-6\%at. Si a été étudiée par spectrométrie d'électron Auger, entre $1023 \mathrm{~K}$ et $1273 \mathrm{~K}$. Les cinétiques de ségrégation du soufre ont été étudiées à $1023 \mathrm{~K}, 1073 \mathrm{~K}$ et $1123 \mathrm{~K}$ et ont permis d'évaluer sa fraction atomique de volume à $3,3 \mathrm{ppm}$ at. L'évolution à l'équilibre des taux de couverture du soufre et du silicium montre que le silicium ne reste en surface qu'au-delà de $1123 \mathrm{~K}$ lorsque la concentration du soufre en surface décroît. Ce phénomène a été interprété par le modèle de Guttmann relatif aux solutions ternaires de substitution en tenant compte d'une énergie d'interaction répulsive entre le soufre et le silicium en surface comme dans la matrice de fer. Les valeurs de l'énergie de ségrégation du soufre dans l'alliage Fe-6\%at. Si et de l'énergie de répulsion en surface entre le soufre et le silicium ont été trouvées respectivement égales à $136 \mathrm{~kJ} / \mathrm{mole}$ et $75,2 \mathrm{~kJ} / \mathrm{mole}$ par ajustement des courbes expérimentales et théoriques.
\end{abstract}

Abstract. - Sulphur and silicon surface segregation was studied by Auger spectroscopy between $1023 \mathrm{~K}$ and $1273 \mathrm{~K}$ on the (100) face of a Fe-6\%at. Si single crystal. Sulphur segregation kinetics recorded at $1023 \mathrm{~K}$, $1073 \mathrm{~K}$ and $1123 \mathrm{~K}$ showed that the bulk concentration of this element decreased from 3.3 to 2.6 p.p.m. at. due to ion etching of the sulphur covered surface. The variation of $\mathrm{Si}$ and $\mathrm{S}$ coverages at equilibrium showed that $\mathrm{Si}$ remains on the surface only above $1123 \mathrm{~K}$ when the sulphur coverage decreases. This phenomenon was interpreted with Guttmann's model concerning ternary substitution solutions, taking into account repulsive interactions between $\mathrm{Si}$ and $\mathrm{S}$ as well at the surface as in the bulk. The repulsion energy between $\mathrm{Si}$ and $\mathrm{S}$ at the surface determined in such a way was : $75.2 \mathrm{~kJ} / \mathrm{mole}$ and the sulphur segregation energy in the $\mathrm{Fe}-\mathrm{Si}$ alloy was found equal to : $136 \mathrm{~kJ} / \mathrm{mole}$.

\section{Introduction.}

Le soufre est une impureté qui entre dans la composition des métaux et alliages métalliques. Lors des traitements thermiques, il ségrège fortement à la surface et aux joints de grains et peut avoir une incidence déterminante sur le comportement des matériaux à l'égard des différentes sollicitations chimiques et mécaniques ainsi que sur la ségrégation des autres éléments [1-3].

De nombreux travaux ont été consacrés à la ségrégation du soufre aux joints de grains et à la surface d'alliages et de métaux [4-6]. Plus particulièrement, la ségrégation du soufre à la surface d'alliages $\mathrm{Fe}-\mathrm{Si}$ a été étudiée de façon qualitative par différents auteurs [7-9].
C'est ainsi que McMahon et al. [7] ont constaté sur un échantillon polycristallin de $\mathrm{Fe}-6 \%$ at. Si contenant les impuretés $\mathrm{C}, \mathrm{S}, \mathrm{B}, \mathrm{N}$, que le soufre commence à ségréger au-delà de $973 \mathrm{~K}$ et déplace toutes les impuretés présentes en surface en dessous de cette température. Toutefois l'influence de cet élément sur la ségrégation du silicium n'a pas été étudiée par ces auteurs.

D'autre part De Rugy [9] a étudié par spectrométrie Auger et diffraction d'électrons lents la ségrégation de Si sur la face (100) d'un alliage de même composition. Il a montré que le silicium forme une couche ordonnée $\mathrm{c}(2 \times 2)$ à saturation et que son énergie de ségrégation est égale à $\Delta G_{\mathrm{Si}}=48$ $0,015 T \mathrm{~kJ} / \mathrm{mole}$. Par contre l'influence du soufre sur la ségrégation du silicium n'a été abordée que 
sommairement dans ce travail. Nous avons donc repris cette étude, par spectrométrie Auger en portant notre attention sur les phénomènes de compétition et les interactions qui peuvent exister entre ces deux éléments après ségrégation.

\section{Echantillon et méthode expérimentale.}

2.1 EChANTILlon ET APPAREILlaGE. - L'échantillon, un alliage Fe-6\%at. Si de haute pureté, est une plaquette de $5 \mathrm{~mm}$ de diamètre et de $0,4 \mathrm{~mm}$ d'épaisseur. Il a été découpé par électroérosion dans un cylindre monocristallin suivant le plan (100).

Après polissage au papier abrasif et à l'alumine, l'échantillon est introduit dans la chambre d'analyse. L'étude a été faite par spectrométrie Auger avec un analyseur de type C.M.A., l'énergie primaire des électrons étant égale à $2500 \mathrm{eV}$ et la tension de modulation à $1,4 \mathrm{~V}$ pic à pic.

Les recuits sont obtenus à l'aide d'un porte-échantillon chauffant comprenant un dispositif de chauffage par bombardement électronique en continu qui permet l'enregistrement des spectres à la température considérée. Sa lecture est assurée à l'aide d'un thermocouple $\mathrm{Pt}-18 \% \mathrm{Rh}$ fixé à la surface de l'échantillon et qui donne la température de celui-ci à $1 \mathrm{~K}$ près. Enfin, le nettoyage de la surface est obtenu par bombardement ionique à l'argon.

2.2 MÉThodes DE MESURES. - Les mesures de cinétique de ségrégation du soufre ont été effectuées après nettoyage de la surface par bombardement aux ions d'argons. L'échantillon étant chauffé et maintenu à température voulue, les spectres Auger de la surface ont été ensuite enregistrés à intervalles de temps réguliers de 30 min jusqu'à l'équilibre que l'on suppose atteint lorsque le pic LMM du soufre reste invariant.

Par ailleurs, les mesures à l'équilibre ont été effectuées après saturation de la surface en soufre à $1023 \mathrm{~K}$. La température est ensuite augmentée lentement par paliers de 30 à $50 \mathrm{~K}$ jusqu'à $1273 \mathrm{~K}$, puis refroidi dans les mêmes conditions afin de rendre compte de la réversibilité de la ségrégation du soufre et du silicium.

Pour nous affranchir des variations d'intensité du faisceau incident, nous avons mesuré les rapports :

et

$$
R_{\mathrm{S}}=H_{\mathrm{S}}(150) / H_{\mathrm{Fe}}(703)
$$

$$
R_{\mathrm{Si}}=H_{\mathrm{Si}}(92) / H_{\mathrm{Fe}}(703)
$$

où $H_{\mathrm{S}}(150), H_{\mathrm{Si}}(92)$ et $H_{\mathrm{Fe}}(703)$ sont les hauteurs respectives du pic LMM à $150 \mathrm{eV}$ du soufre, du pic LMM à $92 \mathrm{eV}$ du silicium et du pic LMM à $703 \mathrm{eV}$ du fer.
Pour les cinétiques de ségrégation, les taux de couverture $\theta_{i}$ sont alors évalués en prenant

$$
\theta_{i}=R_{i} /\left(R_{i}\right)_{\mathrm{eq}}
$$

où $\left(R_{i}\right)_{\mathrm{eq}}$ est le rapport à l'équilibre de l'élément considéré et dépend de la température.

Pour les équilibres de ségrégation, les taux de couverture $\theta_{i}^{\prime}$ sont évalués en prenant $\theta_{i}^{\prime}=$ $R_{i} /\left(R_{i}\right)_{\text {sat }}$ où $\left(R_{i}\right)_{\text {sat }}$ est le rapport correspondant à la surface saturée c'est-à-dire recouverte d'une demimonocouche.

\section{Résultats expérimentaux.}

3.1 DÉTERMINATION DE LA CONCENTRATION VOLUMIQUE DU SOUFRE. - Lorsqu'on chauffe sous vide à une température donnée la surface de Fe-Si nettoyée préalablement par bombardement ionique à l'argon, on constate d'abord la ségrégation du silicium du fait de sa concentration élevée suivie de celle du soufre, et on atteint finalement un équilibre entre ces deux ségrégations compétitives.

Pour interpréter l'équilibre de ségrégation soufresilicium, il est utile de connaître la concentration en volume du soufre en plus de celle du silicium. Ne disposant pas de méthode d'analyse suffisamment sensible, nous avons déterminé cette concentration à partir des cinétiques de ségrégation du soufre que nous avons enregistrées successivement à $1023 \mathrm{~K}$, $1073 \mathrm{~K}$ et $1123 \mathrm{~K}$.

La figure 1 montre ces cinétiques : les variations des taux de couverture $\theta_{\mathrm{S}}$ du soufre à $1023 \mathrm{~K}$, $1073 \mathrm{~K}$ et $1123 \mathrm{~K}$ en fonction de la racine carrée du temps sont respectivement les droites 1,2 et 3 . Les pentes $\theta_{\mathrm{S}} / \sqrt{t}$ augmentent avec la température.

Rawlands et al. [10] et Hofmann et al. [11] pour les systèmes binaires ainsi que Guttmann et al. [12] pour les systèmes ternaires ont montré que la cinétique de ségrégation d'une impureté possédant une forte énergie de ségrégation (cas du soufre) est limitée par la diffusion en volume, et peut être décrite par la loi de Crank :

$$
X^{\prime}(t)=2 X \sqrt{D t} / d \pi
$$

où $X$ et $X^{\prime}$ sont les fractions atomiques de volume et de surface du soufre.

$d=1,43 \AA$ est la distance entre plans atomiques parallèles à la surface.

$D$ est le coefficient de diffusion volumique donné par une loi d'Arrhénius en fonction de l'énergie d'activation de diffusion $Q$ et du facteur de fréquence $D_{0}$ :

$$
D=D_{0} \exp (-Q / R T)
$$

où $Q=207,7 \mathrm{~kJ} / \mathrm{mole}$ et $D_{0}=2,68 \mathrm{~cm}^{2} / \mathrm{s}$ pour le soufre dans Fe-6\%Si d'après Ainslie et al. [13]. Le 


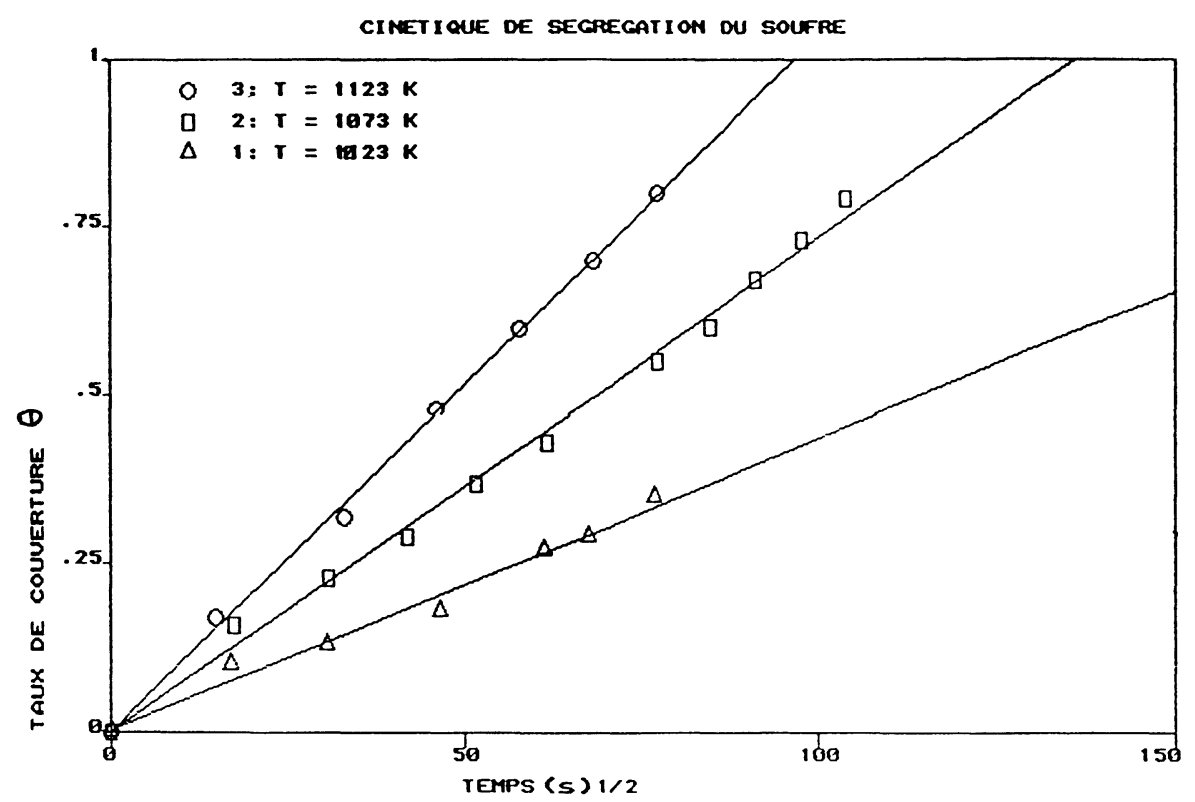

Fig. 1. - Cinétiques de ségrégation du soufre à $1023 \mathrm{~K}, 1073 \mathrm{~K}$ et $1123 \mathrm{~K}$.

[Surface segregation kinetics of sulphur at $1023 \mathrm{~K}, 1073 \mathrm{~K}$ and $1223 \mathrm{~K}$.]

soufre formant à saturation une demi-monocouche atomique, il résulte que $X^{\prime}=\theta_{\mathrm{S}} / 2$.

Connaissant le coefficient de diffusion aux différentes températures nous pouvons, en utilisant les pentes des droites correspondantes, déterminer les concentrations volumiques du soufre de l'échantillon pour chaque cinétique.

Les concentrations ainsi déduites des cinétiques à $1023 \mathrm{~K}, 1073 \mathrm{~K}$ et $1123 \mathrm{~K}$ sont égales respectivement à 3,3, 3,0 et 2,6 ppm. La diminution moyenne d'une cinétique à l'autre est de $0,35 \mathrm{ppm}$. Cet appauvrissement de l'échantillon en soufre s'explique par l'élimination d'une demi-monocouche de soufre en surface par les bombardements ioniques successifs. On peut estimer par ailleurs cet appauvrissement, en tenant compte de l'épaisseur de l'échantillon, $0,4 \mathrm{~mm}$, qui compte donc $2,8 \times 10^{6}$ couches atomiques. Pour obtenir une demi-monocouche de soufre en surface, il faut donc appauvrir l'échantillon de $0,35 \mathrm{ppm}$ en volume.

\subsection{EQUILIBRE DE SÉGRÉGATION SOUFRE-SILI- CIUM.}

3.2.1 Résultats expérimentaux. - Les courbes de la figure 2 montrent l'évolution des taux de couverture du soufre et du silicium à l'équilibre, entre $1073 \mathrm{~K}$ et $1298 \mathrm{~K}$, pour les concentrations en volume du soufre égales respectivement à 3,$3 ; 3,0$ et 2,6 ppm at. (paragraphe 3.1). Notons que les courbes obtenues lors du chauffage et du refroidissement sont superposables et que le phénomène de ségrégation $\mathrm{du}$ soufre et du silicium est donc réversible. Il en résulte donc que les phénomènes d'évaporation peuvent être négligés à ces températures.

D'une manière générale, ces courbes montrent qu'aux températures inférieures à $1073 \mathrm{~K}$, le soufre empêche le silicium de ségréger du fait de son énergie de ségrégation plus élevée. Le silicium ne ségrège en surface qu'à partir du moment où le soufre se redissout dans le volume.

De plus, cette redissolution se fait à des températures de plus en plus basses lorsque la concentration volumique du soufre diminue. La concentration en soufre de départ étant faible (3,3 ppm at.), on observe que lorsque celle-ci diminue de 0,35 ppm environ, les courbes se déplacent de façon sensible vers les basses températures. Enfin d'après Fiedler [19], la solubilité limite du soufre dans l'alliage Fe$6 \%$ at. Si à 1073 K (40 ppm) est bien supérieure à la concentration en volume que nous avons trouvée.

Nous avons interprété les résultats expérimentaux concernant l'équilibre du soufre et du silicium par le modèle de Guttmann [14] relatif aux solutions ternaires de substitution avec compétition sur les sites et interaction entre ces éléments.

3.2.2 Equations de Guttmann. - Les équations reliant les taux de couverture à l'équilibre du soufre $\theta_{\mathrm{S}}$ et du silicium $\theta_{\mathrm{Si}}$ à leurs fractions atomiques de volume $X_{\mathrm{S}}$ et $X_{\mathrm{Si}}$ sont alors les suivantes :

$$
\begin{aligned}
\frac{\theta_{\mathrm{Si}}}{1-\theta_{\mathrm{Si}}-\theta_{\mathrm{S}}} & =\frac{X_{\mathrm{Si}}}{1-X_{\mathrm{S}}-X_{\mathrm{Si}}} \exp \frac{\Delta G_{\mathrm{Si}}}{R T} \\
\frac{\theta_{\mathrm{S}}}{1-\theta_{\mathrm{Si}}-\theta_{\mathrm{S}}} & =\frac{X_{\mathrm{S}}}{1-X_{\mathrm{S}}-X_{\mathrm{Si}}} \exp \frac{\Delta G_{\mathrm{S}}}{R T}
\end{aligned}
$$




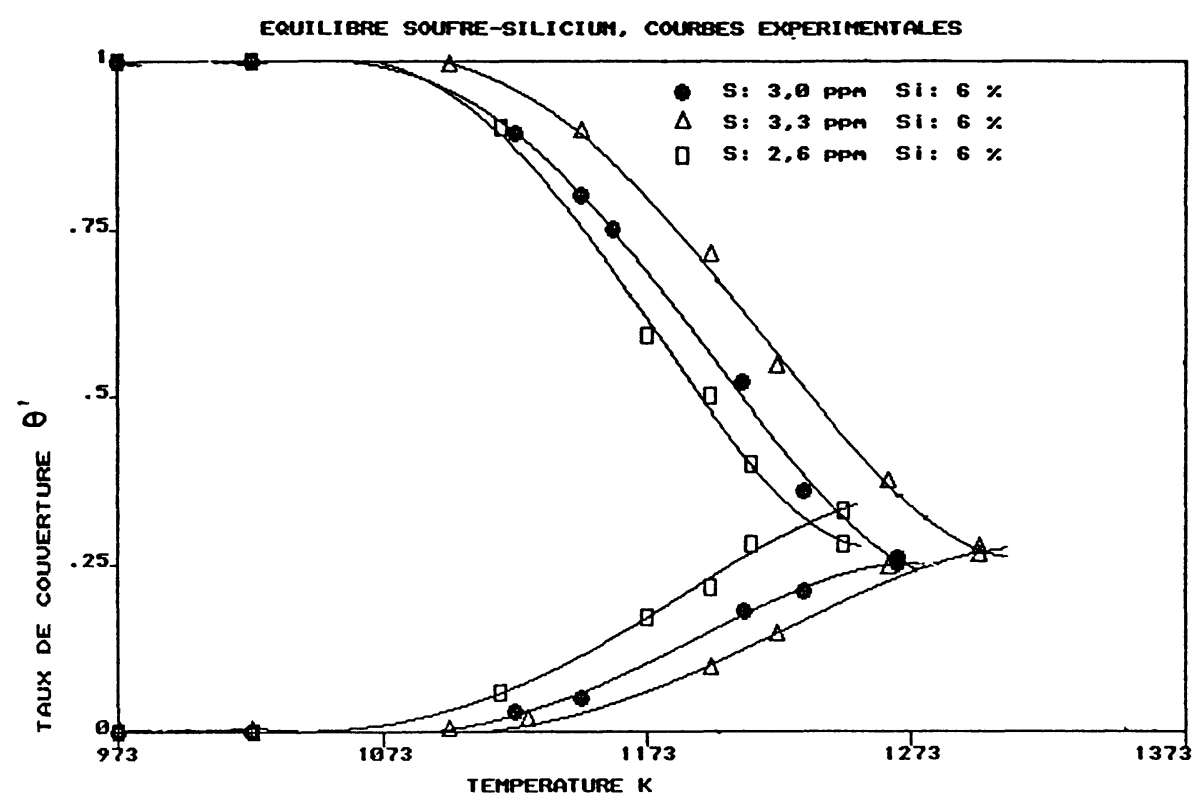

Fig. 2. - Courbes de ségrégation à l'équilibre du soufre et du silicium pour différentes concentrations volumiques en soufre : 3,3 ppm (1), 3,0 ppm (2), 2,6 ppm (3).

[Equilibrium segregation curves of sulphur and silicon for several bulk sulphur concentration : $3.3 \mathrm{ppm}$ (1), $3.0 \mathrm{ppm}$ (2), $2.6 \mathrm{ppm}$ (3).]

où $\Delta G_{\mathrm{S}, \mathrm{Si}}$ sont respectivement les enthalpies libres de ségrégation du soufre et du silicium dans l'alliage $\mathrm{Fe}-\mathrm{Si}$. Ces grandeurs dépendent des enthalpies libres $\Delta G_{\mathrm{S}, \mathrm{Si}}^{0}$ de ces éléments dans les systèmes binaires respectifs $\mathrm{Fe}-\mathrm{S}$ et $\mathrm{Fe}-\mathrm{Si}$, suivant les relations :

$$
\begin{aligned}
\Delta G_{\mathrm{S}} & =\Delta G_{\mathrm{S}}^{0}-\alpha X_{\mathrm{Si}}+\alpha^{\prime} X_{\mathrm{Si}}^{\prime} \\
= & \Delta G_{(\mathrm{Fe}-\mathrm{Si}) \mathrm{S}}^{0}+\alpha^{\prime} \theta_{\mathrm{Si}} / 2 \\
\Delta G_{\mathrm{Si}} & =\Delta G_{\mathrm{Si}}^{0}+\alpha^{\prime} X_{\mathrm{S}}^{\prime} \\
& =\Delta G_{\mathrm{Si}}^{0}+\alpha^{\prime} \theta_{\mathrm{S}} / 2 .
\end{aligned}
$$

Les termes $\alpha^{\prime}$ et $\alpha$ sont les énergies d'interaction entre le soufre et le silicium, respectivement à la surface et dans le volume. La grandeur $\Delta G_{(\mathrm{Fe}-\mathrm{Si}) \mathrm{S}}^{0}=$ $\Delta G_{\mathrm{S}}^{0}-\alpha X_{\mathrm{Si}}$ est l'énergie de ségrégation du soufre dans l'alliage $\mathrm{Fe}-\mathrm{Si}$ quand $\theta_{\mathrm{Si}}=0$. Le soufre et le silicium formant à saturation une demi-monocouche atomique, leurs fractions atomiques respectives sont alors $X_{\mathrm{S}}^{\prime}=\theta_{\mathrm{S}} / 2$ et $X_{\mathrm{Si}}^{\prime}=\theta_{\mathrm{Si}} / 2$. Notons que dans l'équation (5) le terme $\alpha X_{\mathrm{S}}$ a été négligé du fait de la faible valeur de $X_{\mathrm{S}}$.

3.2.3 Détermination de $\Delta G_{\mathrm{S}}^{0}$ et $\alpha^{\prime}$. - Pour pouvoir calculer les taux de couverture $\theta_{\mathrm{S}}$ et $\theta_{\mathrm{Si}}$ à l'aide des équations (3)-(5), il est nécessaire de connaître les valeurs de $\Delta G_{\mathrm{Si}}^{0}, \Delta G_{\mathrm{S}}^{0}, \alpha$ et $\alpha^{\prime}$ en plus des concentrations du soufre et du silicium.

L'énergie de ségrégation du silicium dans l'alliage Fe-6\%at. Si a été déterminée par De Rugy [9], $\Delta G_{\mathrm{Si}}^{0}=48-0,015 T \mathrm{~kJ} / \mathrm{mole}$. Par ailleurs, nous avons calculé la valeur du coefficient d'interaction volumique entre le soufre et le silicium $\alpha=$ $133 \mathrm{~kJ} / \mathrm{mole}$ à l'aide des courbes de solubilité limite du soufre dans Fe-Si $[13,19]$.

$\mathrm{Du}$ fait que l'augmentation de l'enthalpie de ségrégation du soufre $\Delta G_{(\mathrm{Fe}-\mathrm{Si}) \mathrm{S}}^{0}$ entraîne essentiellement un décalage des courbes d'équilibre vers les hautes températures alors que l'augmentation de l'énergie de répulsion $\alpha^{\prime}$ modifie surtout les pentes de ces courbes, il résulte la possibilité de déterminer ces deux grandeurs indépendamment l'une de l'autre par ajustement des courbes calculées (Fig. 3) aux courbes expérimentales (Fig. 2).

On trouve ainsi que l'énergie de répulsion $\alpha^{\prime}$ est égale à $75,2 \mathrm{~kJ} / \mathrm{mole}$ et que l'enthalpie libre de ségrégation du soufre $\Delta G_{(\mathrm{Fe}-\mathrm{Si}) \mathrm{s}}^{0}$ est égale à $136 \mathrm{~kJ} / \mathrm{mole}$.

De la valeur de $\Delta G_{(\mathrm{Fe}-\mathrm{Si}) \mathrm{S}}^{0}$ ainsi trouvée, on déduit à l'aide de l'équation (4) que l'enthalpie libre de ségrégation du soufre dans le fer pur $\Delta G_{\mathrm{S}}^{0}$ est égale à $128 \mathrm{~kJ} / \mathrm{mole}$.

Notons que la valeur $\alpha^{\prime}=75 \mathrm{~kJ} / \mathrm{mole}$ est très voisine de $\alpha / 2$ alors que ce rapport est de $1 / 3$ dans le cas du système Fe-Si-C [15]. Il est difficile de déterminer a priori le rapport entre les valeurs de $\alpha^{\prime}$ et $\alpha$, en raison notamment de la difficulté d'évaluer la coordination des atomes de surface. Mais la valeur de l'énergie d'interaction de surface est généralement inférieure à celle du volume $[15,16]$.

La valeur de l'énergie de ségrégation du soufre 


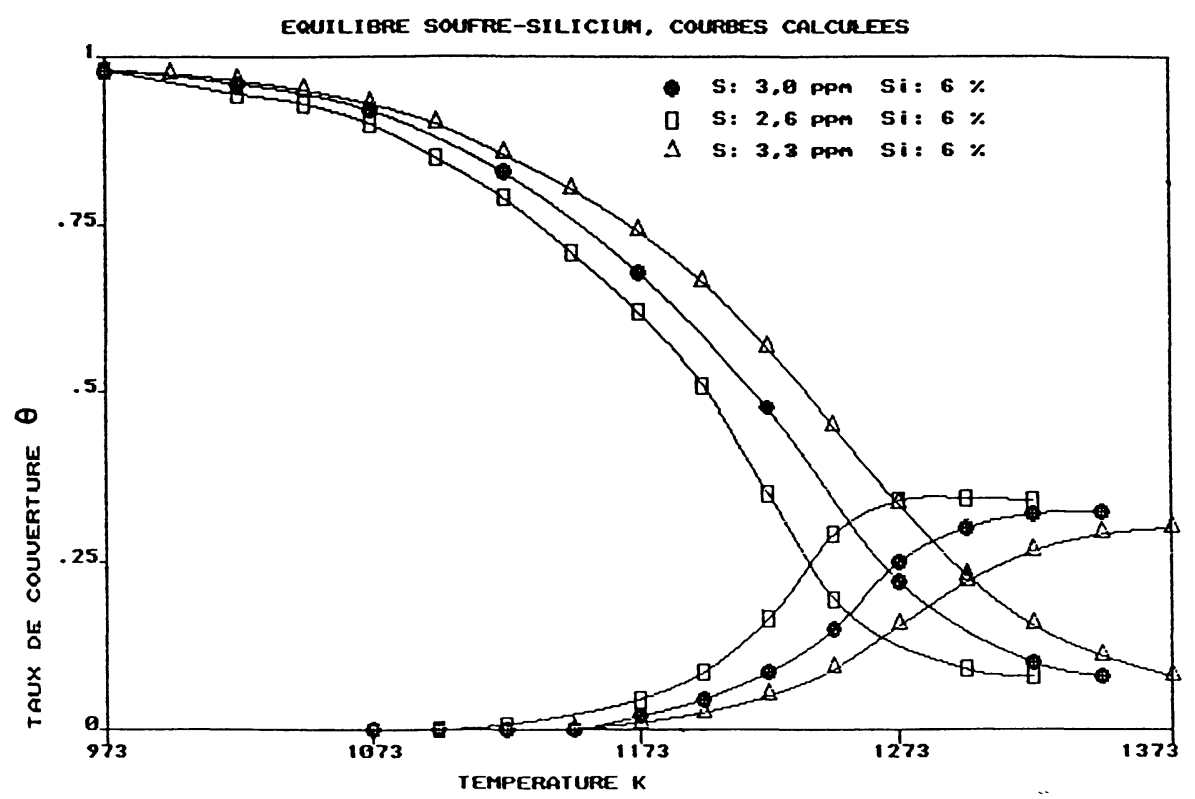

Fig. 3. - Courbes calculées à l'aide du formalisme de Guttmann correspondant aux courbes expérimentales de la figure 2 .

[Calculated curves with Guttmann's formalism corresponding to the experimental curves of figure 2.]

dans le fer pur que nous avons trouvée est inférieure à celle donnée par Grabke et al. [17] (165 kJ/mole). Elle satisfait par contre le critère de Hondros et Seah [18] selon lequel l'énergie de ségrégation d'un élément est supérieure de quelques kilojoules par mole à son énergie de solution. En effet, cette énergie de solution est égale à $120 \mathrm{~kJ} / \mathrm{mole}$ d'après Ainslie et al. [13] entre $923 \mathrm{~K}$ et $1193 \mathrm{~K}$.

\section{Conclusion.}

Nous avons montré que les mesures de cinétique de ségrégation permettent de déterminer avec précision la concentration volumique d'une impureté comme le soufre, lorsqu'on connaît son coefficient de diffusion dans la matrice de l'échantillon. C'est ainsi que la concentration volumique de notre échantillon a été trouvée égale à $3,3 \mathrm{ppm}$ at. au début de nos mesures et qu'elle diminuait de $0,35 \mathrm{ppm}$ environ après chaque bombardement ionique.
D'autre part, les résultats expérimentaux concernant l'équilibre de ségrégation du soufre et du silicium sur la face (100) d'un alliage Fe-6\%at. Si ont été interprétés par le modèle de Guttman relatif aux solutions ternaires de substitution avec compétition sur les sites en considérant une interaction répulsive entre le silicium et le soufre aussi bien en surface que dans le volume. Nous avons ainsi pu déterminer l'enthalpie libre de ségrégation du soufre dans le fer pur $\Delta G_{\mathrm{S}}^{0}$ ainsi que l'énergie d'interaction $\alpha^{\prime}$ entre le soufre et le silicium à la surface.

L'enthalpie libre de ségrégation du soufre dans le fer $\Delta G_{\mathrm{S}}^{0}=128 \mathrm{~kJ} / \mathrm{mole}$ obtenue est très voisine de l'énergie de solution du soufre dans le fer et répond de ce fait au critère de Hondros et Seah [18] qui relie la ségrégation à la solubilité limite.

Par ailleurs, l'énergie de répulsion en surface $\alpha^{\prime}$ entre le soufre et le silicium trouvée égale à $75 \mathrm{~kJ} / \mathrm{mole}$ est très voisine de la moitié de l'énergie de répulsion entre ces mêmes éléments dans le volume.

\section{Bibliographie}

[1] Madon, R. J. and Shaw, H., Catal. Rev. 15 (1977) 69.

[2] Oudar, J., Mater. Sci. Eng. 42 (1980) 401.

[3] Latanisson, R. M., Gastine, O. H. and Compeau, C. R., Symp. on Environment Sensitive Fracture of Engineering materials, Fall Am. Inst. Min., Metall., Pet. Eng., Meet. (1977) pp. 24-26.
[4] Humbert, P. and Mosser, A., Surf. Sci. 126 (1983) 708.

[5] Larere, A., Guttmann, M., Dumoulin, P. and Roques-Carmes, C., Acta Metall. 30 (1982) 685.

[6] Lea, C. and SeaH, M. P., Surf. Sci. 53 (1975) 272. 
[7] Marchut, L. and MCMahon, C. J., Jr., Metall. Trans. 124 (1981) 1136.

[8] Bezuidenhout, F., Du Plessis, J. and Vilijoen, P. E., J. Vac. Sci. Technol. A 2 (1984) 148.

[9] De RugY, thèse de Docteur-Ingénieur, Paris (1982).

[10] Rawlands, G. and Woodruff, D. P., Philos. Mag. 40 (1979) 459.

[11] Hofmann, S. and ERlewein, J., Surf. Sci. 77 (1978) 591.

[12] Guttmann, M., Dumoulin, Ph. and Waymann, N., Metall. Trans., 13 (1982) 1963.

[13] Ainslie, N. G. and Seybolt, A., J. Iron Steel Inst. 194 (1960) 341.
[14] Guttmann, M., Surf. Sci. 53 (1975) 213.

[15] Essouni, M. et Mosser, A., Revue Phys. Appl. 21 (1986) 257.

[16] Dumoulin, Ph. and Guttmann, M., Mater. Sci. Eng. 42 (1980) 249.

[17] Grabke, H. J., Petersen, E. M. and Paulitschke, W., Trans. R. Soc. London Ser. A 295 (1980) 128.

[18] Hondros, E. D. and SeaH, M. P., Metall. Trans. 8A (1977) 1363.

[19] Fiedler, H. C., Trans. Metall. Soc. AIME 239 (1967) 260. 